\title{
Validation of reference genes for quantitative real-time PCR studies in the dentate gyrus after experimental febrile seizures
}

\author{
Ann Swijsen ${ }^{1}$, Katherine Nelissen ${ }^{1}$, Daniel Janssen ${ }^{1}$, Jean-Michel Rigo ${ }^{1 *+}$ and Govert Hoogland ${ }^{2 \dagger}$
}

\begin{abstract}
Background: Quantitative real-time PCR (qPCR) is a commonly used technique to quantify gene expression levels. Validated normalization is essential to obtain reliable qPCR data. In that context, normalizing to multiple reference genes has become the most popular method. However, expression of reference genes may vary per tissue type, developmental stage and in response to experimental treatment. It is therefore imperative to determine stable reference genes for a specific sample set and experimental model. The present study was designed to validate potential reference genes in hippocampal tissue from rats that had experienced early-life febrile seizures (FS). To this end, we applied an established model in which FS were evoked by exposing 10-day old rat pups to heated air. One week later, we determined the expression stability of seven frequently used reference genes in the hippocampal dentate gyrus.

Results: Gene expression stability of 185 rRNA, ActB, GusB, Arbp, Tbp, CycA and Rp/13A was tested using geNorm and Normfinder software. The ranking order of reference genes proposed by geNorm was not identical to that suggested by Normfinder. However, both algorithms indicated CycA, Rp/13A and Tbp as the most stable genes, whereas 185 rRNA and ActB were found to be the least stably expressed genes.

Conclusions: Our data demonstrate that the geometric averaging of at least CycA, Rp/13A and Tbp allows reliable interpretation of gene expression data in this experimental set-up. The results also show that ActB and 185 rRNA are not suited as reference genes in this model.
\end{abstract}

Keywords: Reference gene, Quantitative real-time PCR, Febrile seizures, Dentate gyrus

\section{Background}

Febrile seizures (FS) are convulsions associated with fever and occur in $4 \%$ of children between the age of 3 months and 5 years [1,2]. Retrospective studies demonstrate that adult patients with hippocampal sclerosisassociated temporal lobe epilepsy (TLE) have a $40 \%$ incidence of FS, suggesting a causal relationship [2]. Also, experimental FS have a long-lasting effect on hippocampal excitability, resulting in enhanced seizure susceptibility [3-5]. At a cellular level, an altered seizure threshold may come from a change in the expression of proteins that are known to control neuronal excitability

\footnotetext{
*Correspondence: jeanmichel.rigo@uhasselt.be

${ }^{\dagger}$ Equal contributors

'BIOMED Research Institute, Hasselt University and transnational University Limburg, Agoralaan Bld C, Diepenbeek 3590, Belgium

Full list of author information is available at the end of the article
}

(for review see [6]). Hence, quantification of the post-FS expression of genes that encode receptors, ion channels, etc. might help elucidating FS-induced epileptogenesis.

Nowadays, quantitative real-time PCR (qPCR) is a commonly used tool to quantify gene expression. An advantage of this highly specific and sensitive technique is that it allows analysis on small amounts of starting material $[7,8]$. However, measured gene expression levels may be confounded by several variables during the multistep procedure of isolating and processing RNA e.g. the amount and quality of starting material, enzymatic efficiency and variability between tissues or cells in overall transcriptional activity [9-11]. Internal reference genes are most frequently used to normalize methodology-induced variations in qPCR studies [9,12]. Until recently, 'housekeeping genes' (HKGs) such as glyceraldehyde-3-phosphate dehydrogenase $(G A P D H)$, 18S subunit ribosomal RNA

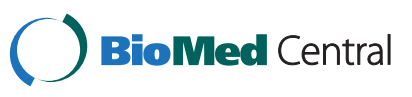


(18S rRNA) or beta-actin (ActB) were commonly used as reference genes, also in neurobiological studies. HKGs are continually read and encode for products that are necessary for the metabolism and existence of a cell. They are supposed to be invariably expressed under different experimental conditions. However, the expression of these classical reference genes may vary per tissue type and developmental stage, and may even vary in response to experimental treatment [13-17]. Hence, validation of suitable reference genes for a specific sample set and experimental model is imperative to obtain consistent gene expression data. In addition, it has been shown that the accuracy of qPCR data further improves when at least three reference genes are used for normalization [9].

Although recent studies have validated reference genes for rat brain tissue in different experimental conditions [18-21], to our knowledge there is thus far no report of validated reference genes in hippocampal tissue from rats that have been exposed to experimental FS. Therefore, we used an established model where FS are evoked by exposing 10-day old rat pups to heated air [22]. One week later, we evaluated the expression stability of seven frequently used reference genes in the hippocampal dentate gyrus (DG). To this end, we used two different mathematical algorithms (geNorm [9] and Normfinder [23] VBA applets for Microsoft Excel) for normalization.

\section{Results}

Seven reference genes (ActB, beta-actin; $C y c A, C y-$ clophylinA; $18 S$ rRNA, $18 \mathrm{~S}$ subunit ribosomal RNA; Rpl13A, Ribosomal protein L13A; Tbp, TATA box binding protein; GusB, beta-glucuronidase; Arbp, Acidic ribosomal phosphoprotein P0) were chosen from literature $[18,24]$ and evaluated for gene expression stability in DG samples from 9 controls, 6 FS- and 7 FS+ rats. Despite its widespread use, GAPDH was not included as a candidate reference gene since other authors showed it to be unstable using the same algorithms applied in the current study $[18,20]$. To avoid coregulation, reference genes were selected from different functional classes. PCR efficiency of the reference genes was situated between 99.96\% and $113.89 \%$ (Table 1).

\section{Cycle threshold values of candidate reference genes}

When all samples were taken together, the reference genes showed cycle threshold (Cq) values varying from a $\mathrm{Cq}$ value of 15.40 for $A r b p$ to 31.22 for Tbp (Additional file 1). With a $\mathrm{Cq} \leq 22.23, A r b p, A c t B, C y c A$ and Rpl13A showed lower Cq values than GusB and Tbp that had a $\mathrm{Cq} \geq 24.42$. $18 S$ rRNA displayed the highest $\mathrm{Cq}$ variability, ranging from 17.91 to 29.75. A similar Cq pattern was observed when $\mathrm{Cq}$ values were calculated per experimental group (Figure 1). Cq standard deviations provide a first idea about the variability in expression, ranking from most to least variably expressed as $18 S r R N A>A c t B>A r b p>$ GusB $>$ Tbp $>$ Rpl13A $>$ CycA.

\section{Validating candidate reference genes}

geNorm is used to determine the average expression stability ( $M$ value), based on the average pairwise variation between a particular gene and all other reference genes in the study. With the exception of $18 S$ rRNA, all genes did show high expression stability, indicated by $M$ values below the default limit of 1.5 suggested by the geNorm

\section{Table 1 Selected reference genes for analysis of expression stability}

\begin{tabular}{|c|c|c|c|}
\hline Gene symbol & Gene function & $\begin{array}{l}\text { Primer sequence }\left(5^{\prime} \rightarrow 3^{\prime}\right)^{a} \text { or } \\
\text { SABiosciences qPCR assay } I D^{b}\end{array}$ & Amplicon length (bp) \\
\hline \multirow[t]{2}{*}{$A c t B$} & Cytoskeletal structural protein & F: TGT CAC CAA CTG GGA CGA TA & 165 \\
\hline & & R: GGG GTG TTG AAG GTC TCA AA & \\
\hline \multirow[t]{2}{*}{$C y C A$} & Serine-threonine phosphatase inhibitor & F: TAT CTG CAC TGC CAA GAC TGA GTG & 126 \\
\hline & & R: CTT CTT GCT GGT CTT GCC ATT CC & \\
\hline \multirow[t]{2}{*}{$18 S$ rRNA } & Ribosomal subunit & F: ACG GAC CAG AGC GAA AGC AT & 310 \\
\hline & & R: TGT CAA TCC TGT CCG TGT CC & \\
\hline \multirow[t]{2}{*}{ Rp/13A } & Structural component of 605 ribosomal subunit & F: GGA TCC CTC CAC CCT ATG ACA & 132 \\
\hline & & R: CTG GTA CTT CCA CCC GAC CTC & \\
\hline \multirow[t]{2}{*}{ Tbp } & General transcription factor & F: TGG GAT TGT ACC ACA GCT CCA & 131 \\
\hline & & R: CTC ATG ATG ACT GCA GCA AAC C & \\
\hline GusB & Exoglycosidase in lysosomes & PPR43194B ${ }^{\mathrm{b}}$ & 137 \\
\hline Arbp & Catalysis of protein synthesis & PPR42394A ${ }^{b}$ & 92 \\
\hline Cnr1 & Endocannabinoid signalling & PPR52793A ${ }^{\mathrm{b}}$ & 156 \\
\hline
\end{tabular}

ActB, beta-actin; CycA, CyclophilinA; 185 rRNA, 18 S subunit ribosomal RNA; Rpl13A, Ribosomal protein L13A; Tbp, TATA box binding protein; GusB, betaglucuronidase; Arbp, Acidic ribosomal phosphoprotein P0; Cnr1, Cannabinoid Type 1 (CB1) receptor. F, forward primer; R, reverse primer. 


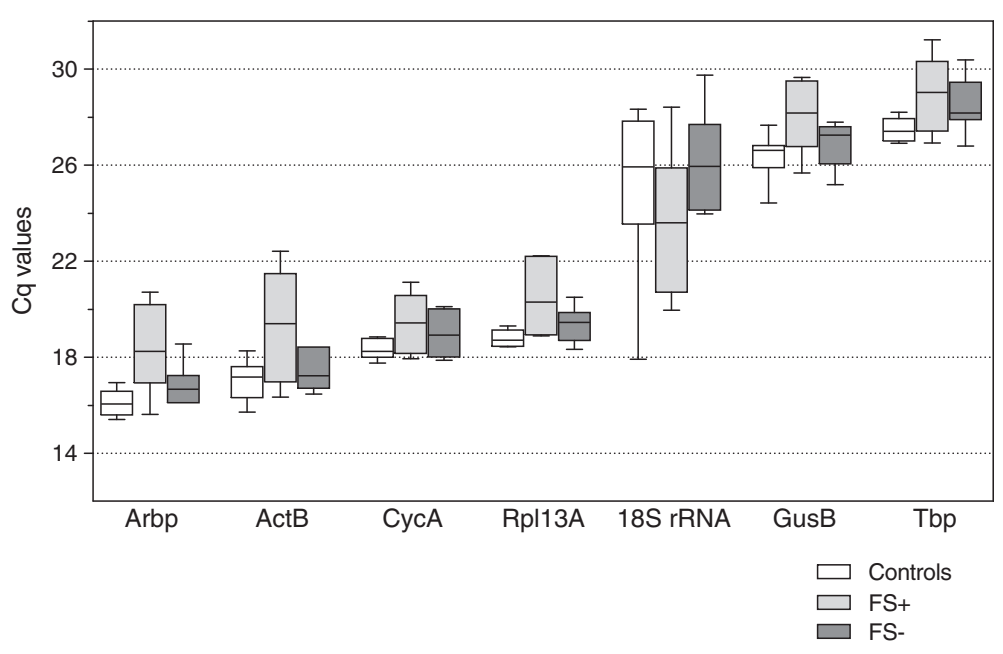

Figure 1 Cycle threshold (Cq) levels of candidate reference genes in each experimental group. Boxes represent lower and upper quartiles with medians, whiskers represent the outer 10\%. Normothermia controls $(n=9)$; FS-, hyperthermia without febrile seizures $(n=6)$; FS+, hyperthermia with febrile seizures $(n=7)$.

software (Figure 2A). Based on the $\mathrm{M}$ values, $\mathrm{CycA}$ and Rpl13A were the most stably expressed genes. It is commonly known that normalization to multiple reference genes is advisable, as the use of a single gene may introduce normalization errors. In that respect, geometric averaging of multiple reference genes is a proven method to calculate an accurate normalization factor [9]. The optimal number of reference genes to be used for normalization can be determined by pairwise variation between two sequential normalization factors $\left(\mathrm{NF}_{\mathrm{n}}\right.$ and $\left.\mathrm{NF}_{\mathrm{n}+1}\right)$, starting with genes with the lowest $M$ value. This analysis learned that for this dataset, the use of five reference genes is optimal when a variation value $\left(\mathrm{V}_{\mathrm{n} / \mathrm{n}+1}\right)<0.15$ is considered as not significantly improving the accuracy (Figure $2 \mathrm{~B}$ ). However, inclusion of the fourth $\left(\mathrm{V}_{3 / 4}=0.176\right)$ and fifth $\left(\mathrm{V}_{4 / 5}=0.153\right)$ most stable gene causes only slight differences in the pairwise variation value (Figure $2 \mathrm{~B}$ ).

The stability ranking of the candidate reference genes determined by geNorm was compared with Microsoft Excel-based applet termed Normfinder. This algorithm provides a stability value for each candidate reference gene and ranks the genes according to their expression stability in a given sample set and experimental design. Normfinder also identified Rpl13A as one of the most stably expressed genes, and $18 S$ rRNA as the least stable gene (Figure $3 \mathrm{~A}$ ). Yet, Tbp was identified as the most stably expressed gene. This algorithm enables to calculate an Acc.SD, which is an indicator of the optimal number of reference genes necessary to obtain an accurate normalization factor. By this approach, we found that an Acc.SD of 0.119 using one gene could be lowered to 0.054 when six genes were included (Figure 3B). Most of this decrease in Acc.SD was attributable to the first three genes $($ Acc.SD $=0.072)$.
The ranking order of reference genes proposed by geNorm was not identical to that suggested by Normfinder. However, both algorithms indicated $C y c A, R p l 13 A$ and $T b p$ as the most stable genes, whereas $18 S$ rRNA and $A c t B$ were found to be the least stably expressed genes (Table 2).

\section{Influence of different normalization approaches on the expression profile of a gene of interest}

To demonstrate the importance of choosing sufficient and stably expressed reference genes, we normalized the expression of the cannabinoid type 1 receptor gene Cnr1 to different normalization factors. This gene of interest was chosen because it was previously shown that hippocampal protein levels of this receptor are significantly increased one week after experimental FS [25]. Here, we first normalized Cnrl to the geometric average of the three reference genes $(C y c A, R p l 13 A$ and $T b p)$ that were indicated as most stably expressed by geNorm and Normfinder analysis. This resulted in a significant upregulation of Cnr1 in FS + rats, compared to controls (Figure 4A). Inclusion of the fourth most stable gene suggested by geNorm, Arbp, in the normalization factor did not change Cnr1 expression levels (Figure 4B). However, the significance of Cnr1 upregulation disappeared when the signal was normalized to the two commonly used reference genes $18 S$ rRNA and $A c t B$, which were identified as the most unstable genes by both algorithms (Figure 4C). This normalization strategy also caused a strong increase in the standard error.

\section{Discussion}

Validated normalization is crucial to obtain reproducible qPCR data of genes of interest. In this context, normalizing to internal reference genes has become the most 


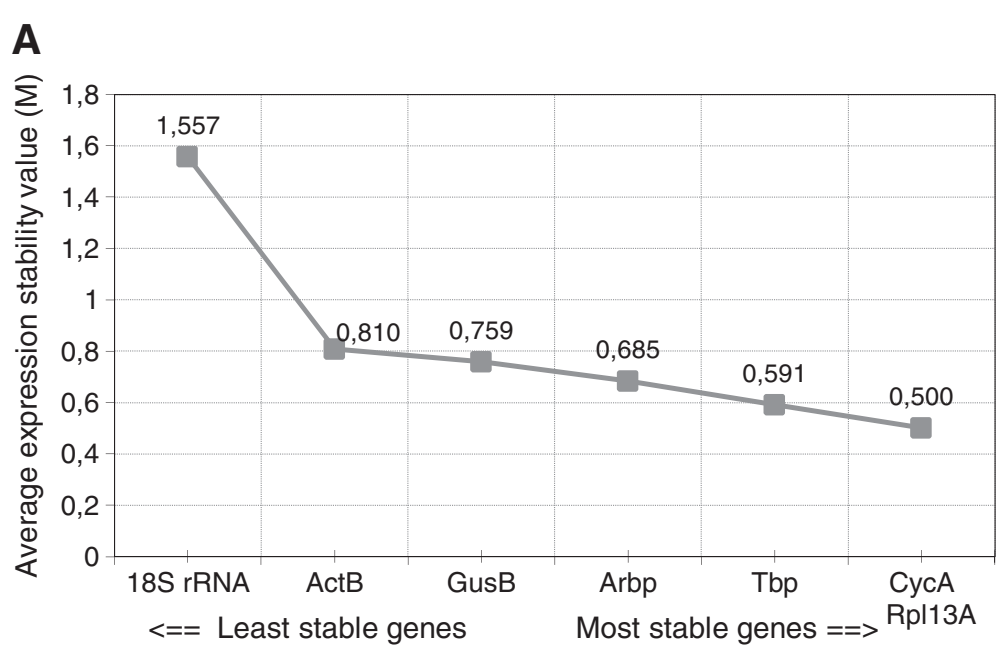

B

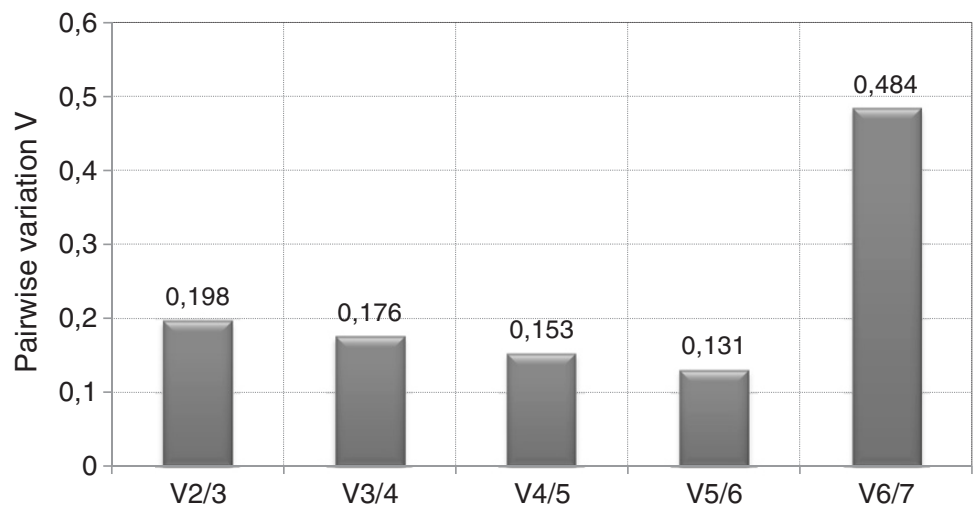

Figure 2 Evaluation of candidate reference genes using geNorm analysis software. A: Average expression stability measure (M) of reference genes in the total sample set $(n=22)$, analyzed by stepwise exclusion of the least stable reference gene. $\mathbf{B}$ : Determination of the optimal number of reference genes for normalization by means of pair-wise variation $\left(V_{n / n+1}\right)$ analysis. Every bar represents the pairwise variation $(V)$ in normalization accuracy when stepwise adding reference genes according to the ranking in $\mathbf{A}$.

popular method to control for experimental errors introduced by the multitude of steps in this analysis. Several studies point out that the expression of reference genes may vary under different experimental conditions [13-17]. This implies the necessity of validating these genes in each new experimental setup.

To our knowledge, this is the first study that describes the stability of $18 S$ rRNA, ActB, GusB, Arbp, Tbp, CycA and Rpl13A in the DG of rats one week after FS. Both, geNorm and Normfinder were used to rank the analyzed reference genes by their expression stability. This rank order differed slightly between both methods, probably because both tools are based on different mathematical models. Other studies have also described similar ranking discrepancies between geNorm and Normfinder $[18,24]$. Interestingly though, both programs agreed on the three most stably expressed genes, being $C y c A$, Tbp and Rpl13A. These converging results stress the significance of including these genes in the normalization factor. In addition, both programs also agreed on $A c t B$ and $18 S$ rRNA as the least stably expressed genes. Comparison of these data with those of recent studies revealed similarities and differences. For instance, Bonefeld et al. [20] validated eight reference genes in rat hippocampal tissue and also identified $C y c A$ and $R p l 13 A$ as the most stably expressed genes and $A c t B$ and $18 S$ $r R N A$ as the least stable genes. Also Pernot et al. [18] found that $C y c A$ and $T b p$ were stably expressed in hippocampus samples from a mouse model of TLE, obtained across different phases of the disease. However, in contrast to our study they also observed a stable $A c t B$ expression. This discrepancy emphasizes the importance of validating reference genes in each experimental model.

Accurate normalization requires inclusion of multiple reference genes. Geometric averaging of the most stable reference genes is a validated method to obtain a reliable normalization factor [9]. Based on a cut-off value of 1.5, 

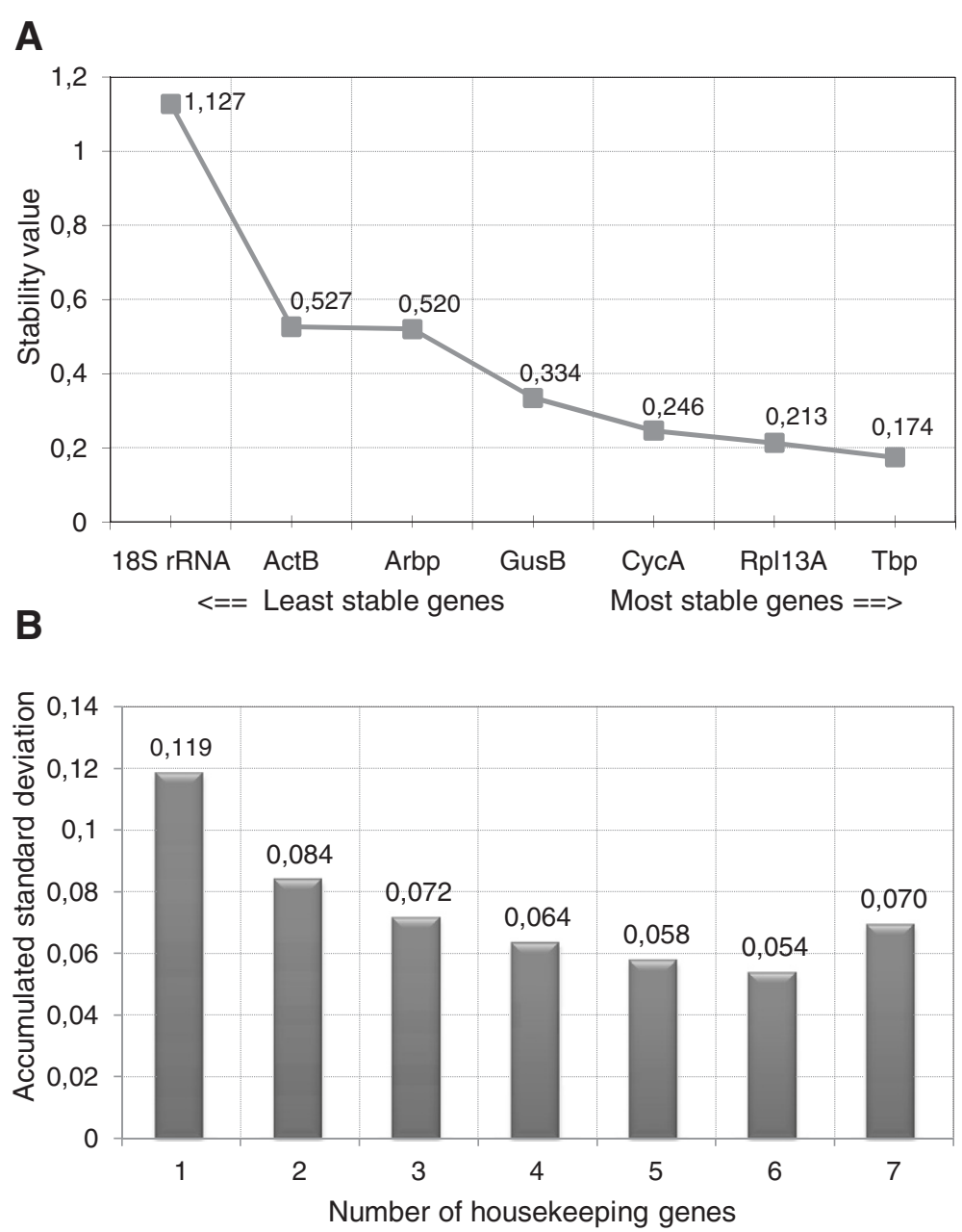

Figure 3 Evaluation of candidate reference genes using Normfinder analysis software. A: Stability values of each reference gene in the total sample set $(n=22)$. B: Determination of the optimal number of reference genes for normalization using the accumulated standard deviation (Acc. SD).

geNorm indicated that the normalization factor should be based on five reference genes. However, according to the geNorm manual, this cut-off value can be set differently. geNorm calculates the optimal number of reference genes by pairwise variation analysis. In that respect, the trend of changing $\mathrm{V}$ values after adding additional genes can be

Table 2 Ranking of reference genes based on the expression stability evaluated by geNorm and Normfinder

\begin{tabular}{cll}
\hline Ranking order & geNorm & Normfinder \\
\hline $\mathbf{1}$ & CycA - Rp/13A & Tbp \\
$\mathbf{2}$ & & Rp/13A \\
$\mathbf{3}$ & Tbp & CycA \\
$\mathbf{4}$ & Arbp & GusB \\
$\mathbf{5}$ & GusB & Arbp \\
$\mathbf{6}$ & ActB & ActB \\
$\mathbf{7}$ & 18S rRNA & $18 S$ rRNA \\
\hline
\end{tabular}

used to obtain an estimate of the number of genes that should be included in the normalization factor. Determination of the optimal number of reference genes is always a trade-off between accuracy and practical considerations, but a minimum of three most stable reference genes is generally recommended [9]. As indicated in Figure 2B, a pairwise variation of 0.198 was observed after adding the third most stable gene. Inclusion of the fourth or fifth most stable gene influenced only slightly the pairwise variation. The high $V_{6 / 7}$ is caused by the high average $M$ value of $18 S$ rRNA, indicating that this gene is highly variably expressed under the present experimental conditions. The Acc.SD calculated by Normfinder suggested the use of six reference genes, though the additive value of genes four to six is minimal. Considering the pairwise variation values, the Acc.SD, and practical issues such as the available amount of RNA, we conclude that the geometric mean of CycA, Rpl13A and Tbp should be used to obtain an accurate normalization factor in this experimental setup. If the 


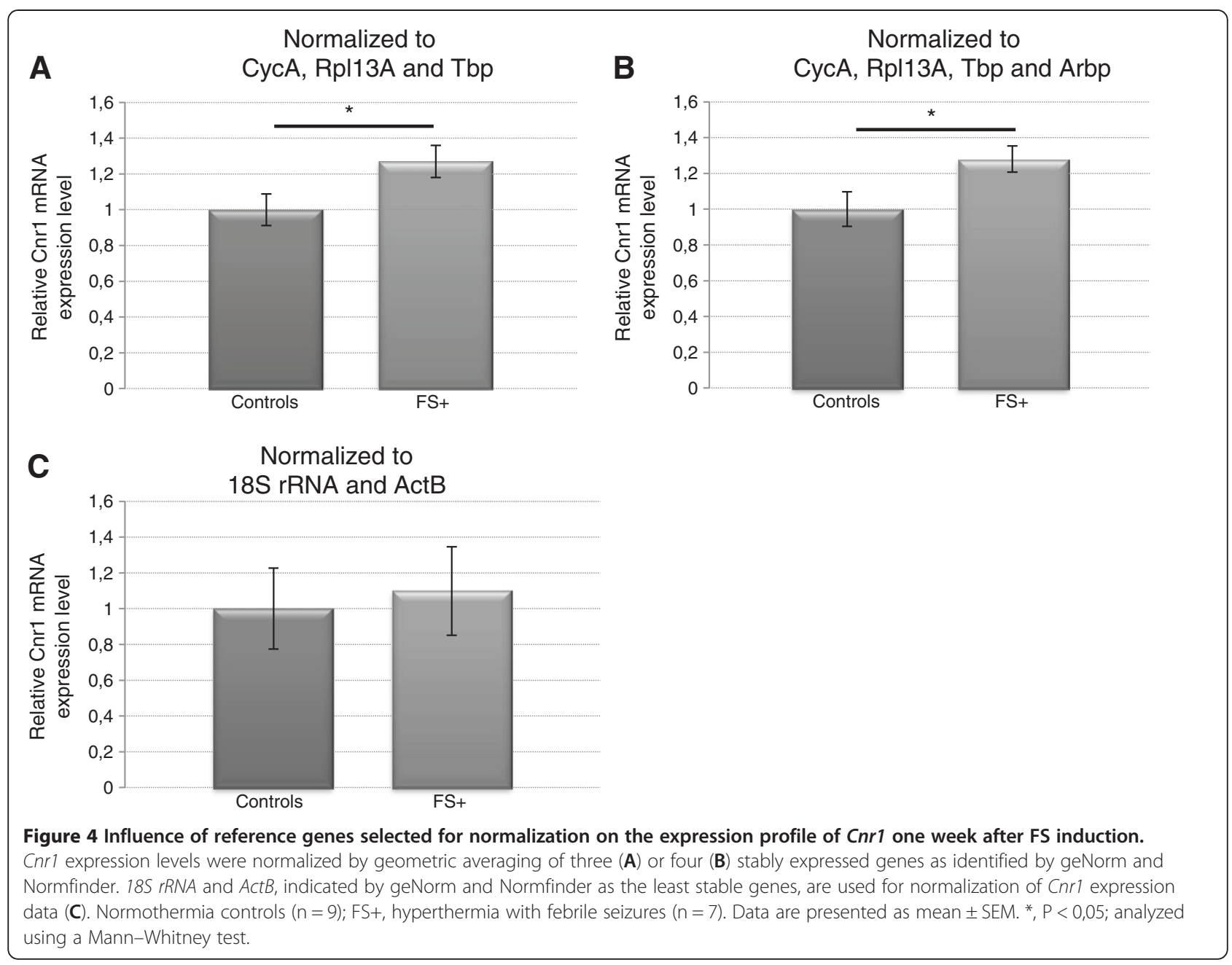

RNA yield allows the inclusion of an extra reference gene, GusB and Arbp may be added to this panel. These data also show that $18 S$ rRNA is unfit as reference gene in this model.

Inclusion of the geNorm/Normfinder selected reference genes in the normalization factor, revealed an increased Cnr1 expression in animals that experienced FS (FS+). This finding is in agreement with quantitative western blot data from Chen et al. [25]. This upregulated Cnr1 disappeared when expression levels were normalized to $A c t B$ and $18 S$ rRNA, underscoring the suggestion that these 'classical' reference genes are not suitable for our experimental setup. In line with this observation, several studies have reported that including $18 S$ rRNA or ActB in the normalization factor altered mRNA expression levels compared to normalization to geNorm proposed genes $[18,20]$. As a possible explanation for erroneous normalization when $18 S$ rRNA is used as reference gene, it has been suggested that this may relate to an imbalance between messenger RNA and ribosomal RNA [17].

\section{Conclusions}

In conclusion, the present study describes the expression stability of seven candidate reference genes in the hippocampal DG of rats, one week after FS. Our results demonstrate that the geometric averaging of at least $C y c A$, Rpl13A and Tbp allows a reliable interpretation of mRNA expression data in this experimental set-up. These data also show that $A c t B$ and $18 S r R N A$ are unfit to serve as reference gene in this model.

\section{Methods}

Induction of febrile seizures and tissue sampling

Litters of 5-10 male Sprague-Dawley rat pups (Harlan, Horst, The Netherlands) were housed with a dam under temperature controlled conditions and $12 \mathrm{~h}$ dark-light cycle with water and food ad libitum. At postnatal day 10, FS were evoked by hyperthermia as described before [26-29]. Briefly, pups were injected subcutaneously with $0.2 \mathrm{ml} 0.9 \% \mathrm{NaCl}$ to prevent dehydration, placed in a perspex cylinder and exposed to a regulated stream of 
heated air. Rectal temperatures were monitored every $2.5 \mathrm{~min}$. A core temperature $>39.5^{\circ} \mathrm{C}$ (usually reached within $5 \mathrm{~min}$ ) indicated the start of a 30 min hyperthermia phase in which the heated air stream was adjusted to maintain a core temperature of $41-42.5^{\circ} \mathrm{C}$. Behavioral seizures occurring during treatment (FS+), were monitored by two observers. These seizures were stereotyped and previously shown to correlate with rhythmic epileptic discharges in the hippocampus [26]. Some rats did not display seizure behavior during the hyperthermia phase (FS-). The hyperthermia phase was terminated by dipping the pup in room temperature water, until the pretreatment body temperature was reached and then returned to the dam. Normothermia control rats underwent the same treatment, except that the stream of air was adjusted to maintain the body temperature that was measured at the start of the experiment $\left(\sim 35^{\circ} \mathrm{C}\right)$. Six to nine days after FS induction, rats were decapitated, brains were rapidly removed from the skull and placed in ice-cold oxygenated $\left(95 \% \mathrm{O}_{2} / 5 \% \mathrm{CO}_{2}\right)$ sucrose-based artificial cerebrospinal fluid (sucrose-aCSF) containing (in $\mathrm{mM}$ ): $210 \mathrm{su}-$ crose, $2.5 \mathrm{KCl}, 26 \mathrm{NaHCO}_{3}, 1.25 \mathrm{NaH}_{2} \mathrm{PO}_{4}, 25$ glucose, 1 $\mathrm{CaCl}_{2}$, and $7 \mathrm{MgSO}_{4}$ (pH 7.4, 340 mOsm). Next, 350- $\mu \mathrm{m}$ -thick coronal slices were cut in ice-cold oxygenated sucrose-aCSF using a vibratome (Microm/Thermo Fisher Scientific, Walldorf, Germany) and DG regions were microdissected from each acute brain slice. DG samples were then quickly frozen in liquid nitrogen and stored at $-80^{\circ} \mathrm{C}$ until RNA isolation. All experiments were approved by the Hasselt University ethics committee for animals.

\section{RNA isolation and CDNA synthesis}

Total RNA was isolated from DG samples using the RNAqueous-Micro kit (Ambion, Lennik, Belgium), according to the manufacturer's protocol. Trace amounts of genomic DNA were removed by DNase I provided with the kit. RNA purity and concentration were checked by optical density, using a NanoDrop ND-1000 spectrophotometer (Thermo Fisher Scientific, Waltham, USA). For cDNA synthesis, total RNA (220 ng) was first incubated for $10 \mathrm{~min}$ at $70^{\circ} \mathrm{C}$ in order to prevent secondary structures, and then reverse transcribed using the Reverse Transcription System (Promega, Leiden, The Netherlands) in a $20 \mu \mathrm{l}$ reaction volume containing 5 $\mathrm{mM} \mathrm{MgCl}_{2}, 1 \times$ Reverse Transcription buffer, $1 \mathrm{mM}$ dNTP mixture, $0.25 \mu \mathrm{g}$ Oligo(dT) 15 primers, $0.25 \mu \mathrm{g}$ hexamer oligonucleotides, $20 \mathrm{U}$ RNase inhibitor and $12.5 \mathrm{U}$ AMV reverse transcriptase, that was first incubated for $60 \mathrm{~min}$ at $42^{\circ} \mathrm{C}$, then for $5 \mathrm{~min}$ at $95^{\circ} \mathrm{C}$ and then at $4^{\circ} \mathrm{C}$. All cDNA samples were stored at $-20^{\circ} \mathrm{C}$ until qPCR analysis.

\section{Quantitative real-time PCR}

qPCR was performed in optical 96-well plates with an ABI PRISM 7500 Fast sequence detection system (Applied Biosystems, Carlsbad, California), and carried out in a $10 \mu \mathrm{l}$ reaction volume containing $5 \mu \mathrm{l}$ RT SYBR green qPCR master mix (SABiosciences/Qiagen, Venlo, The Netherlands), $0.4 \mu \mathrm{M}$ forward and reverse primer (Table 1), and $11 \mathrm{ng}$ cDNA dissolved in nuclease-free water. A no-template control containing nuclease-free water instead of cDNA was included to test for possible contamination of assay reagents. Samples were run in duplicate. PCR conditions comprised a 10 min preincubation at $95^{\circ} \mathrm{C}$, followed by 40 cycles of $15 \mathrm{~s}$ at $95^{\circ} \mathrm{C}$ and $60 \mathrm{~s}$ at $60^{\circ} \mathrm{C}$. Fluorescence was measured at $522 \mathrm{~nm}$ wavelength during each annealing step. Each PCR program was followed by a general dissociation curve protocol to check product specificity. PCR efficiency of the reference genes was determined by a standard curve of cDNA samples according to the MIQE guidelines [30].

\section{Data analysis}

RNA copy numbers were quantified using the comparative $\Delta \Delta \mathrm{Ct}$ method as follows. Raw $\mathrm{Cq}$ values were first transformed to quantities. These raw reference gene quantities that are expressed relative to the sample with the highest quantity, served as data input for geNorm [9] or Normfinder [23]. The geNorm algorithm provides a measure of gene expression stability ( $M$ value) and determines the optimal number of reference genes using pairwise variation $(\mathrm{V})$ analysis. In contrast to geNorm, Normfinder estimates not only the overall expression variation of the candidate reference gene, but also the variation between sample subgroups. The output of Normfinder consists of a stability value based on both intra- and intergroup expression variation. The Accumulated Standard Deviation (Acc.SD), as indicator for the optimal number of reference genes, was determined using GenEx software. For each sample, the normalization factor based on $n$ reference genes was calculated as the geometric average of the $n$ raw reference gene quantities.

Data are presented as mean \pm standard error of the mean (SEM). Statistical analysis was performed using Graphpad Prism5 software. Differences between means were tested using the Mann-Whitney test. A value of $\mathrm{P}<0.05$ was considered as statistically significant.

\section{Additional file}

Additional file 1: Supplementary Table - Cq values of candidate reference genes. Table of $\mathrm{Cq}$ values of all candidate reference genes evaluated in the microdissected dentate gyrus in each experimental condition. Normothermia controls $(n=9)$; FS-, hyperthermia without febrile seizures $(n=6)$; FS+, hyperthermia with febrile seizures $(n=7)$.

\section{Abbreviations}

185 rRNA: 185 subunit ribosomal RNA; ActB: Beta-actin; Arbp: Acidic ribosomal phosphoprotein PO; CyCA: CyclophylinA; $C_{q}$ : Cycle threshold; DG: Dentate gyrus; FS: Febrile seizures; GAPDH: Glyceraldehyde-3-phosphate

dehydrogenase; GusB: Beta-glucuronidase; HKG: Housekeeping gene; 
qPCR: Quantitative real-time PCR; Rp/13A: Ribosomal protein L13A; Tbp: TATA box binding protein; TLE: Temporal Lobe Epilepsy.

\section{Competing interests}

We declare that we have no competing interests.

\section{Authors' contributions}

AS designed and performed the experiments, analyzed and interpreted the data and wrote the manuscript. KN designed the primers and was involved in the analysis and interpretation of the data. DJ helped in generating the animal model and tissue sample collection. JMR and GH designed the research project, supervised the study, were involved in the interpretation of the data and critically revised the manuscript. All authors have read and approved the final manuscript.

\section{Acknowledgements}

We would like to thank Rosette Beenaerts for technical assistance. This research was financially supported by a grant from the transnational University Limburg (tUL) and the Interuniversity Attraction Poles Program (IUAP - Belgian State - Belgian Science Policy).

\section{Author details}

'BIOMED Research Institute, Hasselt University and transnational University Limburg, Agoralaan Bld C, Diepenbeek 3590, Belgium. ${ }^{2}$ Department of Neurosurgery, school of Mental Health and Neurosciences, University Medical Center Maastricht, Maastricht, Netherlands.

Received: 9 July 2012 Accepted: 24 September 2012

Published: 13 December 2012

\section{References}

1. Verity CM, Butler NR, Golding J: Febrile convulsions in a national cohort followed up from birth. I-Prevalence and recurrence in the first five years of life. Br Med J (Clin Res Ed) 1985, 290(6478):1307-1310.

2. Cendes F: Febrile seizures and mesial temporal sclerosis. Curr Opin Neurol 2004, 17(2):161-164.

3. Dube C, Chen K, Eghbal-Ahmadi M, Brunson K, Soltesz I, Baram TZ Prolonged febrile seizures in the immature rat model enhance hippocampal excitability long term. Ann Neurol 2000, 47(3):336-344.

4. Dube C, Richichi C, Bender RA, Chung G, Litt B, Baram TZ: Temporal lobe epilepsy after experimental prolonged febrile seizures: prospective analysis. Brain 2006, 129(Pt 4):911-922.

5. Chen K, Baram TZ, Soltesz I: Febrile seizures in the developing brain result in persistent modification of neuronal excitability in limbic circuits. Nat Med 1999, 5(8):888-894.

6. Dube CM, Brewster AL, Richichi C, Zha Q, Baram TZ: Fever, febrile seizures and epilepsy. Trends Neurosci 2007, 30(10):490-496.

7. Heid CA, Stevens J, Livak KJ, Williams PM: Real time quantitative PCR. Genome Res 1996, 6(10):986-994.

8. Bustin SA: Quantification of mRNA using real-time reverse transcription PCR (RT-PCR): trends and problems. J Mol Endocrinol 2002, 29(1):23-39.

9. Vandesompele J, De Preter K, Pattyn F, Poppe B, Van Roy N, De Paepe A Speleman F: Accurate normalization of real-time quantitative RT-PCR data by geometric averaging of multiple internal control genes. Genome Biol 2002, 3(7):research0034.1-research0034.11.

10. Vermeulen J, De Preter K, Lefever S, Nuytens J, De Vloed F, Derveaux S, Hellemans J, Speleman F, Vandesompele J: Measurable impact of RNA quality on gene expression results from quantitative PCR. Nucleic Acids Res 2011, 39(9):e63.

11. Stahlberg A, Hakansson J, Xian X, Semb H, Kubista M: Properties of the reverse transcription reaction in mRNA quantification. Clin Chem 2004, 50(3):509-515.

12. Huggett J, Dheda K, Bustin S, Zumla A: Real-time RT-PCR normalisation; strategies and considerations. Genes Immun 2005, 6(4):279-284.

13. Derks NM, Muller M, Gaszner B, Tilburg-Ouwens DT, Roubos EW, Kozicz LT: Housekeeping genes revisited: different expressions depending on gender, brain area and stressor. Neuroscience 2008, 156(2):305-309.

14. McCurley AT, Callard GV: Characterization of housekeeping genes in zebrafish: male-female differences and effects of tissue type, developmental stage and chemical treatment. BMC Mol Biol 2008, 9:102.
15. Schmittgen TD, Zakrajsek BA: Effect of experimental treatment on housekeeping gene expression: validation by real-time, quantitative RT-PCR. J Biochem Biophys Methods 2000, 46(1-2):69-81.

16. Deindl E, Boengler K, van Royen N, Schaper W: Differential expression of GAPDH and beta3-actin in growing collateral arteries. Mol Cell Biochem 2002, 236(1-2):139-146.

17. Radonic A, Thulke S, Mackay IM, Landt O, Siegert W, Nitsche A: Guideline to reference gene selection for quantitative real-time PCR. Biochem Biophys Res Commun 2004, 313(4):856-862.

18. Pernot F, Dorandeu F, Beaup C, Peinnequin A: Selection of reference genes for real-time quantitative reverse transcription-polymerase chain reaction in hippocampal structure in a murine model of temporal lobe epilepsy with focal seizures. J Neurosci Res 2010, 88(5):1000-1008.

19. Langnaese K, John R, Schweizer H, Ebmeyer U, Keilhoff G: Selection of reference genes for quantitative real-time $P C R$ in a rat asphyxial cardiac arrest model. BMC Mol Biol 2008, 9:53.

20. Bonefeld BE, Elfving B, Wegener G: Reference genes for normalization: a study of rat brain tissue. Synapse 2008, 62(4):302-309.

21. Harris $J$, Reeves TM, Phillips LL: Injury modality, survival interval, and sample region are critical determinants of qRT-PCR reference gene selection during long-term recovery from brain trauma. J Neurotrauma 2009, 26(10):1669-1681.

22. Jansen JF, Lemmens EM, Strijkers GJ, Prompers JJ, Schijns OE, Kooi ME, Beuls EA, Nicolay K, Backes WH, Hoogland G: Short- and long-term limbic abnormalities after experimental febrile seizures. Neurobiol Dis 2008, 32(2):293-301.

23. Andersen $\mathrm{CL}$, Jensen $J$, Orntoft TF: Normalization of real-time quantitative reverse transcription-PCR data: a model-based variance estimation approach to identify genes suited for normalization, applied to bladder and colon cancer data sets. Cancer Res 2004, 64(15):5245-5250.

24. Nelissen K, Smeets K, Mulder M, Hendriks JJ, Ameloot M: Selection of reference genes for gene expression studies in rat oligodendrocytes using quantitative real time PCR. J Neurosci Methods 2010, 187(1):78-83.

25. Chen K, Ratzliff A, Hilgenberg L, Gulyas A, Freund TF, Smith M, Dinh TP, Piomelli D, Mackie K, Soltesz I: Long-term plasticity of endocannabinoid signaling induced by developmental febrile seizures. Neuron 2003 39(4):599-611.

26. Baram TZ, Gerth A, Schultz L: Febrile seizures: an appropriate-aged model suitable for long-term studies. Brain Res Dev Brain Res 1997, 98(2):265-270.

27. Lemmens EM, Lubbers T, Schijns OE, Beuls EA, Hoogland G: Gender differences in febrile seizure-induced proliferation and survival in the rat dentate gyrus. Epilepsia 2005, 46(10):1603-1612.

28. Lemmens EM, Schijns OE, Beuls EA, Hoogland G: Cytogenesis in the dentate gyrus after neonatal hyperthermia-induced seizures: what becomes of surviving cells? Epilepsia 2008, 49(5):853-860.

29. Swijsen A, Brone B, Rigo JM, Hoogland G: Long-lasting enhancement of $G A B A(A)$ receptor expression in newborn dentate granule cells after early-life febrile seizures. Dev Neurobio/ 2012, Epub ahead of print.

30. Bustin SA, Benes V, Garson JA, Hellemans J, Huggett J, Kubista M, Mueller R, Nolan T, Pfaffl MW, Shipley GL, et al: The MIQE guidelines: minimum information for publication of quantitative real-time PCR experiments. Clin Chem 2009, 55(4):611-622.

\section{doi:10.1186/1756-0500-5-685}

Cite this article as: Swijsen et al:: Validation of reference genes for quantitative real-time PCR studies in the dentate gyrus after experimental febrile seizures. BMC Research Notes 2012 5:685. 